\title{
Holographic Photosensitivity Intensity Dependence of Azobenzene Molecular and Chalcogenide Glassy Films
}

\author{
Peteris Augustovs $^{\mathrm{a}}$, Andris Ozols ${ }^{\mathrm{b}^{*}}$, Elmars Zarins ${ }^{\mathrm{c}}$ and Valdis Kokars ${ }^{\mathrm{d}}$ \\ Faculty of Material Science and Applied Chemistry, Riga Technical University, Paula Valdena iela \\ 3/7, LV-1007, Riga, Latvia \\ apeterisaug@gmail.com, baozols@latnet.Iv, EImars.Zarins@rtu.Iv, Valdis.Kokars@rtu.Iv
}

Keywords: photosensitivity intensity dependence, holographic gratings, azobenzene molecular glassy films, chalcogenide glassy films.

\begin{abstract}
Photosensitivity light intensity dependence of holographic grating recording in three azobenzene molecular glassy films (denoted as WE-3, ZWK-3, ZWK-2TB) and in one chalcogenide glassy film $\mathrm{As}_{40} \mathrm{~S}_{15} \mathrm{Se}_{45}$ (As-S-Se) has been experimentally studied in the intensity range from 0.0034 to $1.13 \mathrm{~W} / \mathrm{cm}^{2}$ at $633 \mathrm{~nm}$. It is found that photosensitivity increases when recording light intensity is increased. The physical reasons are discussed and a simple mathematical model is given explaining the obtained results.
\end{abstract}

\section{Introduction}

Amorphous organic and chalcogenide materials have been widely investigated because of interesting physical phenomena involved and because of many possible applications in optical information storage, nonlinear optics, dynamic holography, holographic optical elements [1-3]. In order to manipulate these materials successfully by light it is necessary to know the effect of light intensity. These measurements are quite time consuming, therefore, this information is not widely available [2,3]. In [2] it was found that steady- state photoinduced anisotropy of azo dye polymer films increases with light intensity increase. Photosensitivity of amorphous chalcogenide $\mathrm{As}_{2} \mathrm{Se}_{3}$ [3] and $\mathrm{As}_{2} \mathrm{~S}_{3}$ [4] films also increases when recording light intensity is increased. In this paper, we present detailed holographic studies of photosensitivity intensity dependence for three azobenzene molecular glassy films (denoted as WE-3, ZWK-3, ZWK-2TB) and one chalcogenide glassy film $\mathrm{As}_{40} \mathrm{~S}_{15} \mathrm{Se}_{45}$ (As-S-Se) . It is found that photosensitivity of all studied samples generally increases when the light sensitivity is increased whereas the physical origin of this fact may be different in different materials. Nevertheless, a simple phenomenological two-state model can be used in all cases to describe qualitatively the main observations.

\section{Experiments and results}

Transmission holographic gratings with the period of $2.0 \mu \mathrm{m}$ were recorded in the above mentioned films by two equally strong symmetrically incident $633 \mathrm{~nm} \mathrm{He}-\mathrm{Ne}$ laser beams with $p-p$ polarizations and self-diffraction efficiency (SDE) was continuously measured according to the method described in [6]. SDE is the ratio of diffracted and one incident beam powers when both recording beams are present. Studied azobenzene molecular glasses were synthesized by our group. They included WE-3 or 2-(4-((4-(bis(2-triphenylsilyloxy)ethyl)amino )phenyl) diazenyl)benzylidene)-1H-indene-1,3(2H)-dione, ZWK-3 or 2-(2-(4-((4-(bis(2trityloxy)ethyl)amino)-phenyl)diazenyl)styryl)-6-styryl-4H-pyran-4-ylidene)- $1 H$-indene-1,3(2H)dione, ZWK-2TB or 2-(2-(4-((4-(bis(2-(trityloxy)ethyl) amino)phenyl)diazenyl)-styryl)-6-tertbutyl-4H-pyran-4-ylidene)-1H-indene-1,3(2H)-dione. About $3 \mu \mathrm{m}$ thick films were spin-coated onto the glass substrates. Their long wavelength absorption maxima were located around 540, 460, and $480 \mathrm{~nm}$, respectively. One chalcogenide glassy film $\mathrm{As}_{40} \mathrm{~S}_{15} \mathrm{Se}_{45}$ (As-S-Se) was studied as well (thickness $4.5 \mu \mathrm{m}$, absorption edge corresponding to $620 \mathrm{~nm}$ ). 
To characterize the film photosensitivity we have determined the recording energies $E=I t$ ( $I$ intensity, $t$ - exposure time) corresponding to $\mathrm{SDE}=0.096 \%$ and $\mathrm{SDE}=0.25 \%$ at certain intensity values, mainly $I=0.011,0.034,0.10,0.32$ and $1.13 \mathrm{~W} / \mathrm{cm}^{2}$. Besides, sensitivities, $S=(S D E)^{0.5} E^{-1}$ corresponding to $\mathrm{SDE}_{\max }$ and the $\mathrm{SDE}_{\max }$ intensity dependences were determined, too. The quantity $S$ is proportional to refractive index change. Experimental results are presented in Figs.1-12.

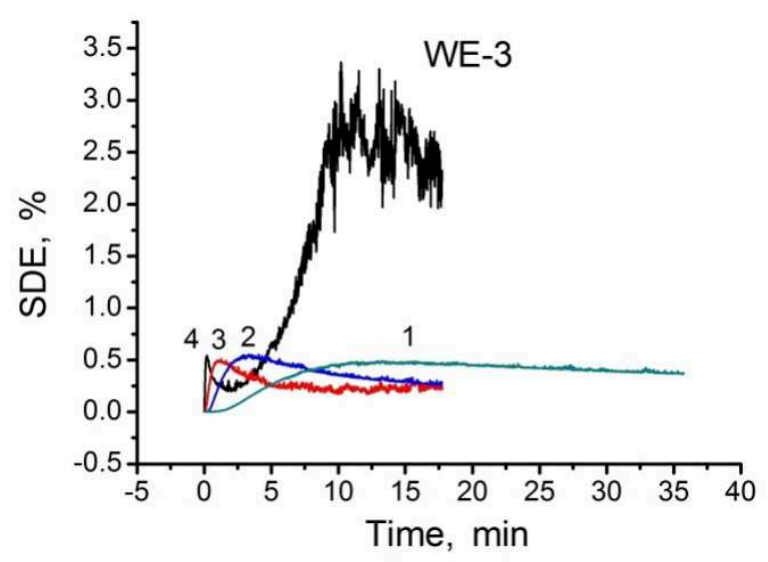

Fig.1. SDE exposure time dependences in the case of sample WE-3 for the following recording intensities: $1-0.034 \mathrm{~W} / \mathrm{cm}^{2}, 2-0.11$ $\mathrm{W} / \mathrm{cm}^{2}, 3-0.34 \mathrm{~W} / \mathrm{cm}^{2}, 4-1.12 \mathrm{~W} / \mathrm{cm}^{2}$.

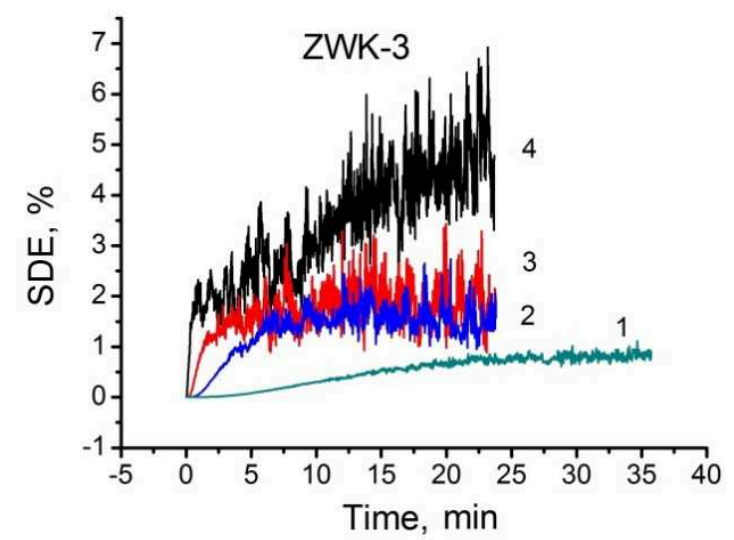

Fig.3. SDE exposure time dependences in the case of sample ZWK-3 for the following recording intensities: $1-0.035 \mathrm{~W} / \mathrm{cm}^{2}, 2-0.11$ $\mathrm{W} / \mathrm{cm}^{2}, 3-0.33 \mathrm{~W} / \mathrm{cm}^{2}, 4-1.16 \mathrm{~W} / \mathrm{cm}^{2}$.

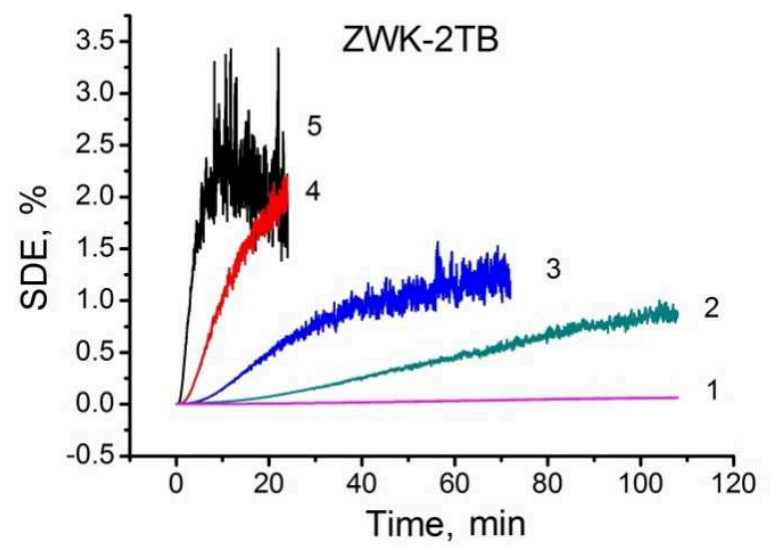

Fig.2. SDE exposure time dependences in the case of sample ZWK-2TB for the following recording intensities:1-0.011 W/ $/ \mathrm{cm}^{2}, 2-0.035$ $\mathrm{W} / \mathrm{cm}^{2}, 3-0.11 \mathrm{~W} / \mathrm{cm}^{2}, 4-0.32 \mathrm{~W} / \mathrm{cm}^{2}, 5-1.13$ $\mathrm{W} / \mathrm{cm}^{2}$.

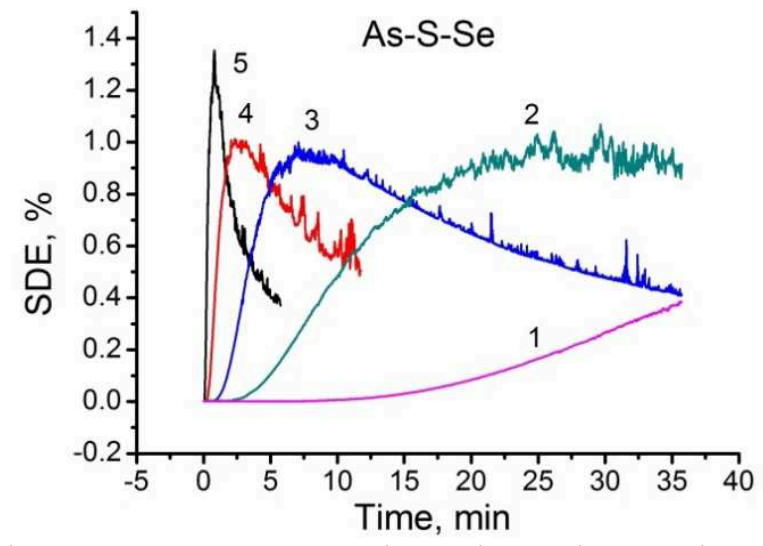

Fig.4. SDE exposure time dependences in the case of sample As-S-Se for the following recording intensities:1-0.011 W/ $/ \mathrm{cm}^{2}, 2-0.034$ $\mathrm{W} / \mathrm{cm}^{2}, 3-0.10 \mathrm{~W} / \mathrm{cm}^{2}, 4-0.32 \mathrm{~W} / \mathrm{cm}^{2}, 5-1.13$ $\mathrm{W} / \mathrm{cm}^{2}$.

One can clearly see from Figs.1-4 that higher intensity enables higher SDE . Further, $E(I)$ dependences are presented for samples WE-3 and As-S-Se, and $S(I)$ dependence for WE-3 (experimental points are connected by straight lines to guide the eye there). For other samples the corresponding dependences are similiar, but they are not presented because of the lack of space.

It is seen from Figs. 5-7 that photosensitivity of studied samples generally increases when the recording light sensitivity is increased. $\mathrm{SDE}_{\max }$ also increases (not shown).

\section{Discussion}

Let us briefly consider the physical recording mechanisms. Azobenzene molecular glasses consist of push-pull type chromophore molecules ( including $\pi$-conjugated donors and acceptors) bound by van der Waals forces [1,2,6,7]. Under the influence of linearly polarized light photo-orientation of chromophores perpendicularly to the light electric vector takes place thus causing the absorption and refractive index changes ( $\Delta \kappa$ and $\Delta n$, respectively). Surface relief gratings also are recorded but 
their contribution in transmission SDE can be neglected [6]. In the case of As-S-Se films photoinduced As-As bond breaking and phonon-assisted As-S and As-Se bond creation takes place [2,5]. Thus in both cases the number $N$ of some active centres per unit volume (oriented chromophores or broken bonds) changes changing also $\Delta \kappa$ and $\Delta n$.

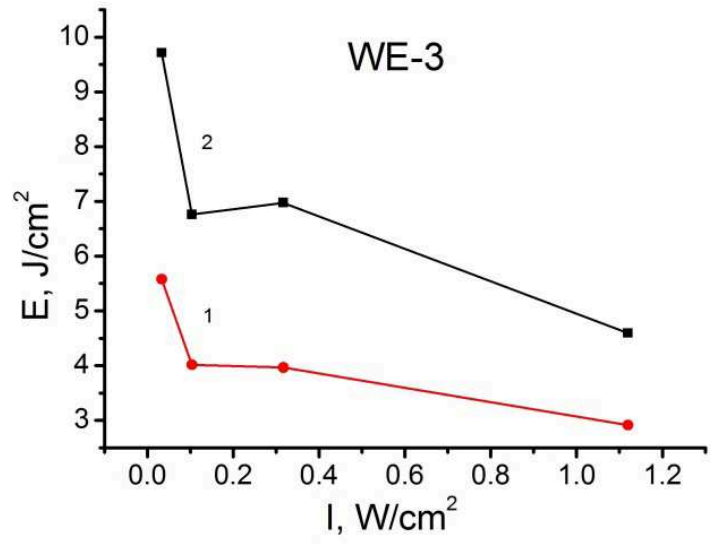

Fig.5. Recording energy intensity dependence in the case of sample WE-3 at fixed SDE values: 1$0.096 \%, 2-0.25 \%$.

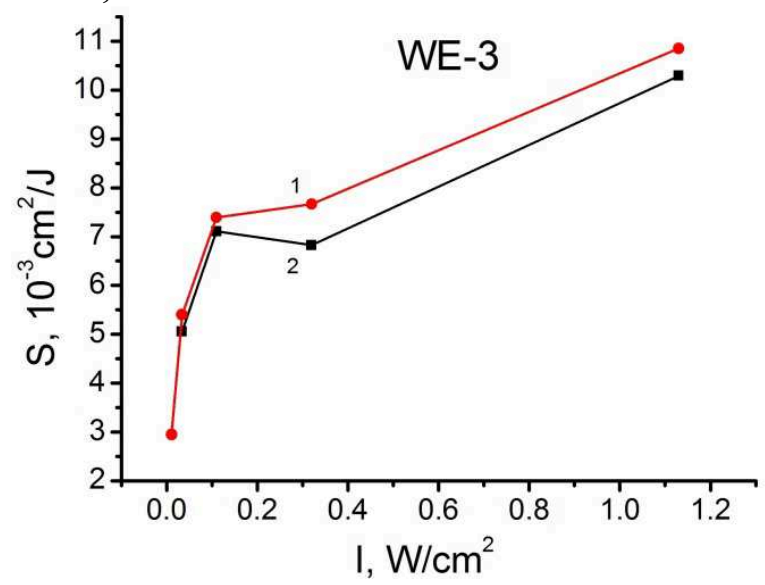

Fig.7. Sensitivity intensity dependence in the case of sample WE-3 at fixed SDE values: 1$0.096 \%, 2-0.25 \%$.

For qualitative explanation of results we consider the recording process as the photo induced transition between the ground state and the excited metastable state accompanied by the back relaxation to the ground state and described by the equation

$\frac{d N}{d t}=a I-\frac{N}{\tau}$

Here $a$ is photo excitation efficiency and $\tau$ is relaxation time. Solving this equation we find the recording energy, $E$ corresponding to a certain $N$ proportional to (SDE) ${ }^{0.5}[6,8]$ :

$$
E=I \tau \ln \frac{a I \tau}{a I \tau-N}
$$


As seen from Fig.8, this formula qualitatively describes the observed experimental dependences (Figs. 5,6). However, it follows from the model that for small enough exposure times compared to the relaxation time, $\tau$, sensitivity

$$
S \propto \frac{N(t \approx 0)}{I t}=a=\text { const }
$$

This is not observed (Fig.7). $S$ increases with $I$. This conclusion holds even if the sensitivity time dependence is taken into account which decreases the $S$ value when approaching the SDE maximum. Therefore, $a$ should also increase with $I$. This really is the case for chalcogenide glasses due to the photo activation of As-As bond breaking by photoinduced nonequilibrium vibrations of atoms [5]. In the case of azobenzene molecular films such $a$ increase can be explained by twophoton excitation of intramolecular charge transfer processes from donors to acceptors leading to chromophore photo-orientation [8]. Thus two-photon processes can explain the observed photosensitivity increase. Yet, the details remain to be found. It should be noted that this model is applicable only up to the maximum of SDE when interference pattern visibility decrease becomes important.

\section{Summary}

Photosensitivity intensity dependence is experimentally studied in azobenzene molecular and chalcogenide glassy films. Its increase with intensity growth is found and explained by a simple two-state model taking into account two-photon processes.

\section{Acknowledgment}

This work has been supported by the European Social Fund within the project No.2013/0028/1DP/1.1.1.2.0/13/APAI/VIAA/054.

\section{References}

[1] Light Wave Manipulation Using Organic Nonlinear Optical Materials. Edited by S.Miyata and H.Sasabe, Taylor\&Francis, London, 2000.

[2] Z.Sekkat, W.Knoll, Photoreactive Organic Thin Films, Orlando, FL ,Elsevier Science, 2002.

[3] K.Schwartz, The Physics of Optical Recording. Berlin, Springer Verlag, 1993.

[4] K.Tanaka, Light intensity dependence of photodarkening in amorphous $\mathrm{As}_{2} \mathrm{~S}_{3}$ films, Thin Solid Films 157 (1988) 35-41.

[5] A.Ozols, Photodarkening nonlinearity in amorphous semiconductor As-S and As-Se films, Latvijas Zinātņu Akadēmijas Vēstis. Fiz. un Tehn. Zin. Sērija 6 (1990) 20-30 (in Russian).

[6] A.Ozols, D.Saharov, V.Kokars, V.Kampars, A.Maleckis, G.Mezinskis, A.Pludons, Holographic recording of surface relief gratings in stilbene azobenzene derivatives at $633 \mathrm{~nm}$, J.of Physics: Conf. Series 249 (2010) 1-8.

[7] G.S.He, L-S Tan, Q.Zheng, and P.N.Prasad, Multiphoton absorbing materials: molecular designs, characterizations, and applications, Chem. Rev. 108(2008) 1245-1330.

[8] A.O.Ozols, K.K. Shvarts, Criteria of medium photosensitivity and hologram recording optimization, Quantum Electronics 9 (1982) 2441-2448 (in Russian). 